\title{
BEST of ASCO 2018
}

\author{
Josef Thaler
}

Received: 25 October 2018 / Accepted: 25 October 2018

(C) Springer-Verlag GmbH Austria, part of Springer Nature 2018
The library of the 2018 annual meeting of the American Society of Clinical Oncology (ASCO) contains 6251 contributions including a range of practice-changing presentations. A selection of major topics from this meeting were presented and discussed in the officially licensed "Best of ASCO" meeting in Vienna on June 22 and 23, 2018. Austrian experts and speakers of this meeting were invited to summarize and discuss the most important and clinically relevant data from the 2018 ASCO meeting in this special memo edition.

To give an impression of the data presented in this memo "BEST of ASCO 2018" series, I will highlight data from three of the seven articles:

Bettina Sonnweber and Ewald Wöll give an update on upper gastrointestinal cancer [1]. Here they report on the PRODIGE24/CCTG PA.6 trial for pancreatic cancer patients in good performance status [2]. It is a multicenter international randomized phase III trial investigating a combined chemotherapy, mFOLFIRINOX, versus standard gemcitabine in patients with resected pancreatic ductal adenocarcinomas (R0 or R1). The primary endpoint was disease-free survival (DFS) and it showed a clear benefit for the mFOLFIRINOX arm (HR of 0.58). The median overall survival revealed a 19-month difference in favor of the mFOLFIRINOX arm, thus representing a new standard of care in selected patients with pancreatic cancer.

Gabriele Gamerith et al. review the highlights from the non-small cell lung cancer (NSCLC) track. Special focus is put on four important phase III trials investi- gating the role of immune checkpoint blockade (ICB) in NCSLC, summarized in Fig. 1 and Table 1 [3] in their article. These studies demonstrate impressive data on ICB combination therapies, thus confirming the major role of ICB in the treatment of patients with NSCLC without genetic alterations.

Erika Richtig gives an overview of the advances in melanoma treatment [4]. For stage III patients, E. Richtig summarizes a discussion on different adjuvant immunotherapy studies. The conclusion was that patients with BRAF wild-type melanoma should receive ICB in the adjuvant setting, BRAF-mutant patients should be offered both options: PD-1 blockade and kinase inhibitors.

For stage IV patients, an update of the Keynote 006 study [5] is presented with a special focus on 4-year survival after a break of 2 years of ICB treatment. Out of 103 patients, who had responded to the ICB treatment for 2 years, the majority of patients maintained their response status during the consecutive treatment-free 2-year period (26/28 patients with complete remission, 56/65 patients with partial remission, and 7/10 patients with stable disease).

I am convinced that the following articles will contribute to the spectrum of your knowledge.

Your sincerely, Josef Thaler

Conflict of interest J. Thaler declares that he has no competing interests.

\footnotetext{
Univ. Prof. Prim. Dr. J. Thaler $(\bowtie)$

Abteilung für Innere Medizin IV, Hämatologie, internistische

Onkologie und Palliativmedizin, Nephrologie und

Dialyse, Klinikum Wels - Grieskirchen, Grieskirchner

Straße 42, 4600 Wels, Austria

josef.thaler@klinikum-wegr.at
} 


\section{editorial}

\section{References}

1. Sonnweber B, Wöll E. ASCO update 2018: upper gastrointestinal cancer. memo. 2018. https://doi.org/10.1007/ s12254-018-0447-4.

2. Conroy T, Hammel P, Hebbar M, et al. Unicancer GI PRODIGE 24/CCTG PA.6 trial: A multicenter international randomized phase III trial of adjuvant mFOLFIRINOX versus gemcitabine in patients with resected pancreatic ductal adenocarcinomas. 2018 ASCO Annual Meeting. J Clin Oncol. 2018;36(suppl):abstr LBA4001.

3. Gamerith G, KocherF, RudzkiJ,PircherA.ASCO2018NSCLC highlights-combination therapy is key. memo. 2018. https://doi.org/10.1007/s12254-018-0444-7.
4. Richtig E. ASCO Congress 2018: melanoma treatment. memo. 2018. https://doi.org/10.1007/s12254-018-0455-4.

5. Long G, et al. ASCO 2018. 2018. https://meetinglibrary. asco.org/record/159075/abstract (Access: 11 November 2018).

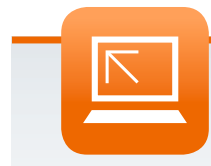

- For latest news from international oncology congresses see: http://www.springermedizin.at/ memo-inoncology 